ANNALES

POLONICI MATHEMATICI

$85.1(2005)$

\title{
A pair of linear functional inequalities and a characterization of $L^{p}$-norm
}

\author{
by Dorota Krassowska and Janusz Matkowski (Zielona Góra)
}

\begin{abstract}
It is shown that, under some general algebraic conditions on fixed real numbers $a, b, \alpha, \beta$, every solution $f: \mathbb{R} \rightarrow \mathbb{R}$ of the system of functional inequalities $f(x+a) \leq f(x)+\alpha, f(x+b) \leq f(x)+\beta$ that is continuous at some point must be a linear function (up to an additive constant). Analogous results for three other similar simultaneous systems are presented. An application to a characterization of $L^{p}$-norm is given.
\end{abstract}

1. Introduction. Every subadditive function $f: \mathbb{R} \rightarrow \mathbb{R}$, that is, such that

$$
f(x+y) \leq f(x)+f(y), \quad x, y \in \mathbb{R},
$$

where $\mathbb{R}$ stands for the set of reals, satisfies the simultaneous system of functional inequalities of additive type:

$$
f(a+x) \leq \alpha+f(x), \quad f(b+x) \leq \beta+f(x), \quad x \in \mathbb{R},
$$

where $a, b \in \mathbb{R}$ are arbitrarily fixed and $\alpha=f(a), \beta=f(b)$. In Section 2 we present some algebraic conditions on $a, b, \alpha, \beta$ under which the only function satisfying this pair of functional inequalities and continuous at some point is $f(x)=\frac{\alpha}{a} x+f(0)$.

In Sections 3, 4 and 5, respectively, we also present analogous conditions for pairs of functional inequalities

$$
\begin{array}{cl}
f(a+x) \leq \alpha f(x), & f(b+x) \leq \beta f(x) ; \\
f(a x) \leq \alpha+f(x), & f(b x) \leq \beta+f(x) ; \\
f(a x) \leq \alpha f(x), & f(b x) \leq \beta f(x) .
\end{array}
$$

The theorems of Sections 2-5 generalize the results of [4], where the corresponding pairs of functional equations were considered (Remark 1). They allow us, in particular, to derive some classical theorems on the Cauchy type functional equation (cf. J. Aczél [1] and M. Kuczma [3]).

2000 Mathematics Subject Classification: Primary 39B72, 26D15; Secondary 46E30.

Key words and phrases: functional inequality, functional equation, theorem of Kronecker, $L^{p}$-norm-like functional, subhomogeneity, characterization of $L^{p}$-norm. 
For a measure space $(\Omega, \Sigma, \mu)$ denote by $\mathbb{S}=\mathbb{S}(\Omega, \Sigma, \mu)$ the linear space of all $\mu$-integrable simple functions $x: \Omega \rightarrow \mathbb{R}$. Let $\phi:(0, \infty) \rightarrow(0, \infty)$ be an arbitrary bijection. As an application, in Section 6 , we give a new characterization of the $L^{p}$-norm with the aid of a rather weak subhomogeneity condition on the $L^{p}$-norm-like functional $\mathbf{p}_{\phi}$,

$$
\mathbf{p}_{\phi}(x):=\left\{\begin{array}{ll}
\phi^{-1}\left(\int_{\Omega_{x}} \phi \circ|x| d \mu\right), & \mu\left(\Omega_{x}\right)>0, \\
0, & \mu\left(\Omega_{x}\right)=0,
\end{array} \quad x \in \mathbb{S},\right.
$$

where $\Omega_{x}:=\{\omega \in \Omega: x(\omega) \neq 0\}$. Let us mention that in A. C. Zaanen [8], W. Wnuk [7], and J. Matkowski [5], the functional $\mathbf{p}_{\phi}$ is assumed to be positively homogeneous.

By $\mathbb{N}, \mathbb{Z}$, and $\mathbb{Q}$ we denote, respectively, the sets of natural, integer, and rational numbers.

\section{Inequalities of additive type}

Theorem 1. Let $a, b, \alpha, \beta \in \mathbb{R}$ be fixed numbers. Suppose that

$$
a<0<b, \quad \frac{b}{a} \notin \mathbb{Q}, \quad \frac{\alpha}{a} \geq \frac{\beta}{b},
$$

and a function $f: \mathbb{R} \rightarrow \mathbb{R}$ is continuous at least at one point.

If $f$ satisfies the pair of functional inequalities

$$
f(a+x) \leq \alpha+f(x), \quad f(b+x) \leq \beta+f(x), \quad x \in \mathbb{R},
$$

then

$$
f(x)=\frac{\alpha}{a} x+f(0), \quad x \in \mathbb{R} .
$$

Proof. From (1), by induction, we obtain

$$
f(m a+x) \leq m \alpha+f(x), \quad f(n b+x) \leq n \beta+f(x), \quad m, n \in \mathbb{N}, x \in \mathbb{R} .
$$

Replacing $x$ by $n b+x$ in the first of these inequalities we hence get

$$
f(m a+n b+x) \leq m \alpha+n \beta+f(x), \quad m, n \in \mathbb{N}, x \in \mathbb{R} .
$$

Since $b / a \notin \mathbb{Q}$, and $a b<0$, the Kronecker theorem (cf. [6]) implies that the set

$$
A=\{m a+n b: m, n \in \mathbb{N}\}
$$

is dense in $\mathbb{R}$. Thus there exist two sequences $\left(m_{k}\right),\left(n_{k}\right)$ of positive integers such that

$$
\lim _{k \rightarrow \infty}\left(m_{k} a+n_{k} b\right)=0
$$

Note that

$$
\lim _{k \rightarrow \infty} m_{k}=\lim _{k \rightarrow \infty} n_{k}=\infty
$$


(otherwise $b / a$ would be rational). Obviously,

$$
\lim _{k \rightarrow \infty} \frac{m_{k} a+n_{k} b}{m_{k}}=0,
$$

and, consequently,

$$
\lim _{k \rightarrow \infty} \frac{n_{k}}{m_{k}}=-\frac{a}{b}
$$

Let $x_{0} \in \mathbb{R}$ be a point of continuity of $f$. From (2) we get

$$
f\left(m_{k} a+n_{k} b+x_{0}\right) \leq m_{k} \alpha+n_{k} \beta+f\left(x_{0}\right), \quad k \in \mathbb{N},
$$

or, equivalently,

$$
\frac{f\left(m_{k} a+n_{k} b+x_{0}\right)}{m_{k}} \leq \alpha+\frac{n_{k}}{m_{k}} \beta+\frac{f\left(x_{0}\right)}{m_{k}}, \quad k \in \mathbb{N} .
$$

Letting $k \rightarrow \infty$, and making use of (3), (4), and the continuity of $f$ at $x_{0}$, we hence get $0 \leq \alpha-\frac{a}{b} \beta$, i.e.

$$
\frac{\beta}{b} \geq \frac{\alpha}{a}
$$

As, by the assumption, the reverse inequality holds true, we have shown that

$$
\frac{\alpha}{a}=\frac{\beta}{b}
$$

Now, setting

$$
p:=\frac{\alpha}{a}=\frac{\beta}{b},
$$

we can write inequality $(2)$ in the form

$$
f(t+x) \leq p t+f(x), \quad t \in A, x \in \mathbb{R} .
$$

Take an arbitrary $x \in \mathbb{R}$. By the density of $A$ there is a sequence $\left(t_{n}\right)$ such that

$$
t_{n} \in A \quad(n \in \mathbb{N}), \quad \lim _{n \rightarrow \infty} t_{n}=x_{0}-x .
$$

From (5) we have

$$
f\left(t_{n}+x\right) \leq p t_{n}+f(x), \quad n \in \mathbb{N} .
$$

Letting $n \rightarrow \infty$, and making use of the continuity of $f$ at $x_{0}$, we obtain

$$
f\left(x_{0}\right) \leq p\left(x_{0}-x\right)+f(x), \quad x \in \mathbb{R} .
$$

To prove the opposite inequality note that replacing $x$ by $x-t$ in (5) we get

$$
f(x) \leq p t+f(x-t), \quad t \in A, x \in \mathbb{R} .
$$

Taking an $x \in \mathbb{R}$, and, by the density of $A$, a sequence $\left(t_{n}\right)$ such that

$$
t_{n} \in A \quad(n \in \mathbb{N}), \quad \lim _{n \rightarrow \infty} t_{n}=x-x_{0},
$$


we hence get

$$
f(x) \leq p t_{n}+f\left(x-t_{n}\right), \quad n \in \mathbb{N} .
$$

Letting $n \rightarrow \infty$, and again making use of the continuity of $f$ at $x_{0}$, we obtain

$$
f(x) \leq p\left(x-x_{0}\right)+f\left(x_{0}\right), \quad x \in \mathbb{R} .
$$

Thus

$$
f(x)=p x+\left(f\left(x_{0}\right)-p x_{0}\right), \quad x \in \mathbb{R},
$$

which was to be shown.

Remark 1. Let $a, b, \alpha, \beta \in \mathbb{R}, a b \neq 0$, be such that $\beta / b=\alpha / a$.

If $b / a \notin \mathbb{Q}$ and a function $f: \mathbb{R} \rightarrow \mathbb{R}$ is continuous at least at one point and satisfies the simultaneous system of functional equations

$$
f(a+x)=\alpha+f(x), \quad f(b+x)=\beta+f(x), \quad x \in \mathbb{R},
$$

then $f(x)=p x+q$ for some $p, q \in \mathbb{R}, x \in \mathbb{R}$ (cf. [4]).

If $b / a \in \mathbb{Q}$ then this system of functional equations reduces to the single functional equation

$$
f(d+x)=\frac{\alpha}{a}+f(x), \quad x \in \mathbb{R},
$$

where $d:=\min \{m a+n b>0: m, n \in \mathbb{N}\}$.

Since the continuous and monotonic solution of this equation depends on an arbitrary function (cf. M. Kuczma [2]), the assumption that $b / a \notin \mathbb{Q}$ in Theorem 1 is essential.

REMARK 2. The assumption $\alpha / a \geq \beta / b$ is essential for the uniqueness of the solution of system (1) in Theorem 1. Indeed, if $\alpha / a<\beta / b$ the set of solutions of (1) is large; for instance the function $f:=\sin$ satisfies (1) for all $a, b \in \mathbb{R}$ and $\alpha, \beta \geq 2$. Moreover every affine function of the form $f(x)=A x+B$ where $B \in \mathbb{R}$ is arbitrary and $\alpha / a \leq A \leq \beta / b$ is a solution of (1).

\section{Inequalities of additive-multiplicative type}

Lemma 1. Let $a, b, \alpha, \beta$ be fixed real numbers such that

$$
a<0<b, \quad \frac{b}{a} \notin \mathbb{Q}, \quad \alpha, \beta>0 .
$$

Suppose that $f: \mathbb{R} \rightarrow \mathbb{R}$ is continuous at least at one point and satisfies the system of functional inequalities

$$
f(a+x) \leq \alpha f(x), \quad f(b+x) \leq \beta f(x), \quad x \in \mathbb{R},
$$

or

$$
f(a+x) \geq \alpha f(x), \quad f(b+x) \geq \beta f(x), \quad x \in \mathbb{R} .
$$

Then $f$ is either positive in $\mathbb{R}$, negative in $\mathbb{R}$, or identically zero. 
Proof. Assume that $f: \mathbb{R} \rightarrow \mathbb{R}$ satisfies (6), $x_{0}$ is a point of continuity of $f$ and $f\left(x_{0}\right)>0$. From (6), by induction, we get

$$
f(m a+n b+x) \leq \alpha^{m} \beta^{n} f(x), \quad m, n \in \mathbb{N}, x \in \mathbb{R} .
$$

Take an arbitrary $x \in \mathbb{R}$. By the density of the set $A=\{m a+n b: m, n \in \mathbb{N}\}$ in $\mathbb{R}$ there exists a sequence $\left(m_{k} a+n_{k} b: k, m_{k}, n_{k} \in \mathbb{N}\right)$ such that

From (8) we have

$$
\lim _{k \rightarrow \infty}\left(m_{k} a+n_{k} b\right)=x_{0}-x .
$$

$$
f\left(m_{k} a+n_{k} b+x\right) \leq \alpha^{m_{k}} \beta^{n_{k}} f(x), \quad m_{k}, n_{k} \in \mathbb{N}, x \in \mathbb{R} .
$$

For $k$ large enough, by the continuity of $f$ at $x_{0}$, the left-hand side of this inequality is positive. It follows that $f$ is positive.

Suppose now that $f\left(x_{0}\right)<0$. Replacing $x$ by $x-(m a+n b)$ in (8) we get

$$
f(x) \leq \alpha^{m} b^{n} f(x-(m a+n b)), \quad m, n \in \mathbb{N}, x \in \mathbb{R} .
$$

Now, similarly to the previous case, fix $x \in \mathbb{R}$ and take a sequence $\left(m_{k} a+\right.$ $n_{k} b: k, m_{k}, n_{k} \in \mathbb{N}$ ) such that

$$
\lim _{k \rightarrow \infty}\left(m_{k} a+n_{k} b\right)=x-x_{0} .
$$

Again by the continuity of $f$ at $x_{0}$, for $k$ large enough, the right-hand side of inequality (9) is negative and hence so is $f(x)$.

If $f\left(x_{0}\right)=0$ an argument analogous to the first step shows that $f(x) \geq 0$ for all $x \in \mathbb{R}$, and a slight modification of the argument of the second step gives the inequality $f(x) \leq 0$ for all $x \in \mathbb{R}$, and, consequently, $f=0$ in $\mathbb{R}$.

To complete the proof it is enough to repeat the same reasoning for system (7).

Theorem 2. Let $a, b \in \mathbb{R}$ and $\alpha, \beta>0$ be fixed numbers such that

$$
a<0<b, \quad \frac{b}{a} \notin \mathbb{Q}, \quad \frac{\log \alpha}{a} \geq \frac{\log \beta}{b} .
$$

Suppose that a function $f: \mathbb{R} \rightarrow \mathbb{R}$ is continuous at least at one point and such that $f(\mathbb{R}) \nsubseteq(-\infty, 0)$. If $f$ satisfies the pair of functional inequalities (6), i.e.

$$
f(a+x) \leq \alpha f(x), \quad f(b+x) \leq \beta f(x), \quad x \in \mathbb{R},
$$

then either $f$ is identically zero in $\mathbb{R}$, or

$$
f(x)=f(0) e^{\frac{\log \alpha}{a} x}, \quad x \in \mathbb{R} .
$$

Proof. Assume that $f$ satisfies (6). By the assumptions and Lemma 1 the function $f$ is either identically zero in $\mathbb{R}$ or positive in $\mathbb{R}$. In the first case there is nothing to prove. In the second case $f$ is positive and the function $g:=\log \circ f$ satisfies the inequalities

$$
g(a+x) \leq \log \alpha+\log f(x), \quad g(b+x) \leq \log \beta+\log f(x), \quad x \in \mathbb{R},
$$

and our theorem results from Theorem 1. 
Obviously, for inequalities (7) an analogous result holds true.

REMARK 3. The assumption $f(\mathbb{R}) \nsubseteq(-\infty, 0)$ in Theorem 2 is essential. To see this, take arbitrary $a, b \in \mathbb{R}$ such that $a<0<b, b / a \notin \mathbb{Q}, \alpha, \beta \in$ $(0,1 / 2)$, and an arbitrary function $f: \mathbb{R} \rightarrow[-2,-1]$. Then for all $x \in \mathbb{R}$,

$$
f(a+x) \leq-1=\frac{1}{2} \cdot(-2) \leq \frac{1}{2} f(x) \leq \alpha f(x)
$$

and similarly, for all $x \in \mathbb{R}$,

$$
f(b+x) \leq \beta f(x),
$$

which proves that $f$ satisfies (6). Since $\log \alpha<0$ and $\log \beta<0$ and $a<0<b$, we have

$$
\frac{\log \alpha}{a}<0<\frac{\log \beta}{b} .
$$

Thus all assumptions of Theorem 2 except the condition $f(\mathbb{R}) \nsubseteq(-\infty, 0)$ are satisfied.

4. Inequalities of multiplicative-additive type. As an easy consequence of Theorem 1 we have

TheOREm 3. Let $a, b, \alpha, \beta$ be fixed real numbers such that

$$
0<a<1<b, \quad \frac{\log b}{\log a} \notin \mathbb{Q}, \quad \frac{\alpha}{\log a} \geq \frac{\beta}{\log b} .
$$

Suppose that a function $f: I \rightarrow \mathbb{R}$ is continuous at least at one point and satisfies the pair of functional inequalities

$$
f(a x) \leq \alpha+f(x), \quad f(b x) \leq \beta+f(x), \quad x \in I,
$$

where either $I=(0, \infty)$ or $I=(-\infty, 0)$.

(i) If $I=(0, \infty)$, then

$$
f(x)=\frac{\alpha}{\log a} \log x+f(1), \quad x>0 .
$$

(ii) If $I=(-\infty, 0)$, then

$$
f(x)=\frac{\alpha}{\log a} \log (-x)+f(-1), \quad x<0 .
$$

Corollary 1. Let $a, b, \alpha, \beta$ satisfy the assumptions of Theorem 3 . If a function $f:(-\infty, 0) \cup(0, \infty) \rightarrow \mathbb{R}$ satisfies the pair of inequalities (10), and in each of the intervals $(-\infty, 0)$ and $(0, \infty)$ there is at least one point of continuity of $f$, then

$$
f(x)= \begin{cases}\frac{\alpha}{\log a} \log x+f(1) & \text { for } x \in(0, \infty), \\ \frac{\alpha}{\log a} \log (-x)+f(-1) & \text { for } x \in(-\infty, 0) .\end{cases}
$$


REMARK 4. Suppose that $a, b, \alpha, \beta$ are fixed real numbers such that $0<a<1<b$ and $\alpha / \log a=\beta / \log b$. Note that if $0 \in I$ then there is no function satisfying (10). Indeed, putting $x=0$ into (10) we get $0 \leq \alpha, 0 \leq \beta$, which contradicts the assumptions.

5. Inequalities of multiplicative type. The following counterpart of Lemma 1 is easy to verify.

Lemma 2. Let $a, b, \alpha, \beta$ be fixed positive real numbers such that

$$
a<1<b, \quad \frac{\log b}{\log a} \notin \mathbb{Q},
$$

and $I=(0, \infty)$ or $I=(-\infty, 0)$. Suppose that $f: I \rightarrow \mathbb{R}$ is continuous at least at one point and satisfies the system of functional inequalities

$$
f(a x) \leq \alpha f(x), \quad f(b x) \leq \beta f(x), \quad x \in I,
$$

or

$$
f(a x) \geq \alpha f(x), \quad f(b x) \geq \beta f(x), \quad x \in I .
$$

Then $f$ is either positive in $I$, negative in I, or identically zero.

Applying Lemma 2 and Theorem 1 we obtain

TheORem 4. Let $a, b, \alpha, \beta$ be fixed positive real numbers such that

$$
a<1<b, \quad \frac{\log b}{\log a} \notin \mathbb{Q}, \quad \frac{\log \alpha}{\log a} \geq \frac{\log \beta}{\log b},
$$

and $I=(0, \infty)$ or $I=(-\infty, 0)$. Suppose that $f: I \rightarrow \mathbb{R}$ is continuous at least at one point, satisfies the pair of functional inequalities

$$
f(a x) \leq \alpha f(x), \quad f(b x) \leq \beta f(x), \quad x \in I,
$$

and $f(I) \nsubseteq(-\infty, 0)$. Then either $f$ is identically zero in $I$ or

(i) in the case $I=(0, \infty)$,

$$
f(x)=f(1) x^{\frac{\log \alpha}{\log a}}, \quad x>0,
$$

(ii) in the case $I=(-\infty, 0)$,

$$
f(x)=f(-1)(-x)^{\frac{\log \alpha}{\log a}}, \quad x<0 .
$$

We omit the formulation of the corresponding result for inequalities (12).

Remark 5. Suppose that $a, b, \alpha, \beta$ are fixed positive real numbers such that $a<1<b$ and $\frac{\log \alpha}{\log a}=\frac{\log \beta}{\log b}$. Note that if $I=\mathbb{R}$ or $I=[0, \infty)$ or $I=(-\infty, 0]$ and $f: I \rightarrow \mathbb{R}$ satisfies $(13)$, then $f(0)=0$. Indeed, by assumptions either $\alpha<1<\beta$ or $\beta<1<\alpha$ and, moreover, $f(0)(1-\alpha) \leq 0$ and $f(0)(1-\beta) \leq 0$. Thus $f(0)=0$. 
Hence we get

REMARK 6. (i) Suppose that $f:[0, \infty) \rightarrow \mathbb{R}$ satisfies (13). If $\left.f\right|_{(0, \infty)}$ and $a, b, \alpha, \beta$ satisfy the assumptions of Theorem 4 , then

$$
f(x)= \begin{cases}f(1) x^{\frac{\log \alpha}{\log a}} & \text { for } x \in(0, \infty), \\ 0 & \text { for } x=0 .\end{cases}
$$

(ii) Suppose that $f:(-\infty, 0] \rightarrow \mathbb{R}$ satisfies (13). If $\left.f\right|_{(-\infty, 0)}$ and $a, b, \alpha, \beta$ satisfy the assumptions of Theorem 4 , then

$$
f(x)= \begin{cases}f(-1)(-x)^{\frac{\log \alpha}{\log a}} & \text { for } x \in(-\infty, 0), \\ 0 & \text { for } x=0 .\end{cases}
$$

Finally, let us record the following

REMARK 7. For obvious reasons the counterparts of Theorems 1-4 for the reverse inequalities remain true.

6. A characterization of $L^{p}$-norm. Recall that A. C. Zaanen [8], for the counting measure space, W. Wnuk [7], assuming the continuity of the function $\phi$, and J. Matkowski [5], assuming much weaker regularity conditions, characterized the $L^{p}$-norm with the aid of the homogeneity of the functional $\mathbf{p}_{\phi}$ (cf. the definition in the Introduction).

As an application of Theorem 4 we present a far-reaching generalization of these results. It turns out that the homogeneity condition can be replaced by an inequality assumed to be satisfied only for two characteristic functions $\chi_{A}, \chi_{B}$ of suitably chosen measurable sets $A$ and $B$.

Theorem 5. Let $(\Omega, \Sigma, \mu)$ be a measure space with two sets $A, B \in \Sigma$ such that

$$
0<\mu(A)<1<\mu(B)<\infty, \quad \frac{\log \mu(B)}{\log \mu(A)} \notin \mathbb{Q} .
$$

Suppose that $\phi:(0, \infty) \rightarrow(0, \infty)$ is a bijection such that $\phi^{-1}$ is continuous at least at one point and

$$
\frac{\log \phi^{-1}(\mu(A) \phi(1))}{\log \mu(A)} \geq \frac{\log \phi^{-1}(\mu(B) \phi(1))}{\log \mu(B)}
$$

If $\mathbf{p}_{\phi}$ satisfies the condition

$$
\mathbf{p}_{\phi}(t x) \leq t \mathbf{p}_{\phi}(x), \quad t>0, x \in\left\{\chi_{A}, \chi_{B}\right\}
$$

then

where

$$
\phi(t)=\phi(1) t^{p}, \quad t>0,
$$

$$
p:=\frac{\log \phi^{-1}(\mu(A) \phi(1))}{\log \mu(A)} .
$$

Moreover, if $p \geq 1$ then $\mathbf{p}_{\phi}$ coincides with the $L^{p}$-norm. 
Proof. Let $a=\mu(A)$ and $b=\mu(B)$. From (15) we obtain

$$
\phi^{-1}(a \phi(t)) \leq t \phi^{-1}(a \phi(1)), \quad \phi^{-1}(b \phi(t)) \leq t \phi^{-1}(b \phi(1)), \quad t>0,
$$

which with $\alpha:=\phi^{-1}(a \phi(1))$ and $\beta:=\phi^{-1}(b \phi(1))$ reduces to the pair of functional inequalities

$$
\phi^{-1}(a \phi(t)) \leq \alpha t, \quad \phi^{-1}(b \phi(t)) \leq \beta t, \quad t>0 .
$$

From the bijectivity of $\phi$, replacing here $t$ by $\phi^{-1}(t)$, we get the equivalent system of inequalities

$$
\phi^{-1}(a t) \leq \alpha \phi^{-1}(t), \quad \phi^{-1}(b t) \leq \beta \phi^{-1}(t), \quad t>0,
$$

which, with $f:=\phi^{-1}$ and $I=(0, \infty)$, takes the form (13). Now our result follows from Theorem 4 .

REMARK 8. Note that (15) is a very weak substitute of the homogeneity of the functional $\mathbf{p}_{\phi}$.

Discussing the assumptions in Theorem 5 , note that the condition: $\frac{\log b}{\log a}$ $\notin \mathbb{Q}$ or $\frac{\log (a+b)}{\log a} \notin \mathbb{Q}$ is not too demanding.

To show that the assumption of the existence of sets $A$ and $B$ with $0<\mu(A)<1<\mu(B)<\infty$ is essential, we indicate some wide classes of non-power functions $\phi$ for which the functional $\mathbf{p}_{\phi}$ satisfies the condition (15), in each of the cases

$$
\begin{array}{ll}
\mu(A) \leq 1 \text { or } \mu(A)=\infty & \text { for every } A \in \Sigma ; \\
\mu(A) \geq 1 \text { or } \mu(A)=0 & \text { for every } A \in \Sigma .
\end{array}
$$

EXAMPle 1 . Let $(\Omega, \Sigma, \mu)$ be a measure space such that $\mu(\Omega) \leq 1$. Put $\delta:=\inf \{\mu(A): A \in \Sigma \wedge \mu(A)>0\}$. Let $\phi:(0, \infty) \rightarrow(0, \infty)$ be an increasing bijection such that the function $(0, \infty) \ni t \mapsto \phi(t) / t$ is non-increasing and $\phi(\delta)=\delta, \phi(1)=1$. Then $\phi(t)=t$ for all $t \in[\delta, 1]$, the function $(0, \infty) \ni t \mapsto \phi^{-1}(t) / t$ is non-decreasing and, therefore, for each $A \in \Sigma$ with $a:=\mu(A)>0$, we have

$$
\begin{aligned}
\mathbf{p}_{\phi}\left(t \chi_{A}\right) & =\phi^{-1}(a \phi(t))=\frac{\phi^{-1}(a \phi(t))}{a \phi(t)} a \phi(t) \\
& \leq \frac{\phi^{-1}(\phi(t))}{\phi(t)} a \phi(t)=t a=t \phi^{-1}(a \phi(1)) \\
& =t \mathbf{p}_{\phi}\left(\chi_{A}\right), \quad t>0 .
\end{aligned}
$$

Thus $\mathbf{p}_{\phi}$ satisfies (15) and $\phi$ is not a power function.

EXAMPle 2 . Let $(\Omega, \Sigma, \mu)$ be a measure space for which $\mu(A) \geq 1$ for every set $A \in \Sigma$ such that $\mu(A)>0$, and there exists $B \in \Sigma$ such that $1<\mu(B)<\infty$. Then $\delta:=\inf \{\mu(A): A \in \Sigma \wedge \mu(A)>0\} \geq 1$. Let $\phi:(0, \infty) \rightarrow(0, \infty)$ be a bijection such that the function $(0, \infty) \ni t \mapsto \phi(t) / t$ 
is non-decreasing and $\phi(1)=1, \phi(\delta)=\delta$. Then $\phi$ is strictly increasing, $\phi(t)=t$ for all $t \in[1, \delta]$, the function $(0, \infty) \ni t \mapsto \phi^{-1}(t) / t$ is nonincreasing, and therefore, in the same way as in the previous example, for all $B \in \Sigma$ such that $0<\mu(B)<\infty$, we have

$$
\mathbf{p}_{\phi}\left(t \chi_{B}\right) \leq t \mathbf{p}_{\phi}\left(\chi_{B}\right), \quad t>0 .
$$

We end our discussion with an example showing that the assumption (14) is indispensable.

EXAMPLE 3 . Let $(\Omega, \Sigma, \mu)$ be an arbitrary measure space, and $f: \mathbb{R} \rightarrow \mathbb{R}$ a bijection such that $f(0)=0$ and $f^{-1}$ is subadditive (for instance, for $f$ one can take the inverse function to $x \mapsto x+|\sin x|)$. Define $\phi:(0, \infty) \rightarrow(0, \infty)$ by $\phi(t)=e^{f(\log t)}$. Then, making use of the definition of $\mathbf{p}_{\phi}$, the subadditivity of $f^{-1}$, and the monotonicity of the exponential function, for all $A \in \Sigma$ with $a:=\mu(A)>0$, we have

$$
\begin{aligned}
\mathbf{p}_{\phi}\left(t \chi_{A}\right) & =\phi^{-1}(a \phi(t))=e^{f^{-1}(\log a+f(\log t))} \\
& \leq e^{f^{-1}(\log a)} e^{f^{-1}(f(\log t))} \\
& =t \phi^{-1}(a)=t \mathbf{p}_{\phi}\left(\chi_{A}\right), \quad t>0
\end{aligned}
$$

Thus $\mathbf{p}_{\phi}$ satisfies the subhomogeneity condition (15) for all functions $\chi_{A}$ (here $\mu(A)$ can be smaller or greater than 1 ). This shows that in Theorem 5 condition (14) cannot be omitted.

Acknowledgements. The authors would like to thank the reviewer for his valuable suggestions.

\section{References}

[1] J. Aczél, Lectures on Functional Equations and their Applications, Academic Press, New York and London, 1966.

[2] M. Kuczma, Functional Equations in a Single Variable, Monografie Mat. 46, PWNPolish Sci. Publ., Warszawa, 1968.

[3] - An Introduction to the Theory of Functional Equations and Inequalities, Cauchy's Equation and Jensen's Inequality, PWN-Polish Sci. Publ. and Silesian University, Warszawa-Kraków-Katowice, 1985.

[4] J. Matkowski, Cauchy functional equation on a restricted domain and commuting functions, in: Iteration Theory and its Functional Equations (Schloss Hofen, 1984), Springer, 1985, 101-106.

[5] -, On a characterization of $L^{p}$-norm, Ann. Polon. Math. 50 (1989), 137-144.

[6] - , The converse of the Minkowski's inequality theorem and its generalization, Proc. Amer. Math. Soc. 109 (1990), 663-675. 
[7] W. Wnuk, Orlicz spaces cannot be normed analogously to $L^{p}$-spaces, Indag. Math. 46 (1984), 357-359.

[8] A. C. Zaanen, Some remarks about the definition of an Orlicz space, in: Lecture Notes in Math. 945, Springer, 1982, 261-268.

Dorota Krassowska

Faculty of Mathematics, Informatics

and Econometry

University of Zielona Góra

PL-65-246 Zielona Góra, Poland

E-mail: D.Krassowska@wmie.uz.zgora.pl
Janusz Matkowski

Faculty of Mathematics, Informatics and Econometry

University of Zielona Góra PL-65-246 Zielona Góra, Poland E-mail: J.Matkowski@im.uz.zgora.pl and Institute of Mathematics Silesian University PL-40-007 Katowice, Poland

Reçu par la Rédaction le 24.7.2003

Révisé le 24.1.2005 